\title{
Massive stars near the Eddington-limit, pulsations \& mass-loss
}

\author{
G. Gräfener \\ Armagh Observatory, College Hill, Armagh, BT61 9DG, United Kingdom
}

\begin{abstract}
Very massive stars (in excess of $\sim 100 M_{\odot}$ ) and massive stars in pre-SN phases at the end of their evolution are continuously approaching the Eddington limit. According to our theoretical predictions their high Eddington factors lead to a peculiar sub-photospheric structure and enhanced mass-loss. Their proximity to the Eddington limit is thus likely the reason why these objects appear as LBVs and WR stars. Here we discuss how our predictions relate to the characteristics of strange-mode pulsations, and how rotating massive stars at low metallicities can produce spectroscopic signatures that have recently been observed in a sample of star-forming galaxies at redshifts $z \approx 2-4$.
\end{abstract}

\section{Introduction}

In this contribution we discuss two processes that are predicted to occur when massive stars are approaching the Eddington limit. A radial inflation of the outer stellar envelope, and strong, Wolf-Rayet (WR) type mass-loss. Both processes are caused by the so-called Fe-opacity peak, i.e. an enhancement of the flux-weighted mean opacity that occurs in a density and temperature regime where the Fe-group elements have very complex electron configurations. We highlight the relation of these processes to stellar pulsations, and their relevance for stellar populations at low metallicity.

\section{Envelope inflation near the Eddington limit}

Figure 1 shows the Hertzsprung-Russell (HR) diagram of Galactic WR stars of the WN subtype from Hamann et al. (2006). The diagram shows two populations of WN stars. A group of extremely luminous H-rich WN stars (WNh stars) near the ZAMS, and a group of less luminous H-poor and H-free WN stars between the ZAMS and the He-ZAMS. Both groups of stars show strong WR-type mass loss. WNh stars are believed to be extremely massive stars $\left(M \gtrsim 100 M_{\odot}\right)$ that are still in the core H-burning phase. They constitute the group of stars with the highest masses known, i.e., the top of the main-sequence (cf., Gräfener \& Hamann 2008; Crowther et al. 2010; Gräfener et al. 2011; Bestenlehner et al. 2014).

Here we discuss the group of hot, H-poor WN stars which consists predominantly of core He-burning stars in a post-RSG or post-LBV phase (e.g., Gräfener et al. 2012b). Hamann et al. (2006) discussed that the majority of such objects would be expected to lie near the He-ZAMS, i.e., at much higher temperatures than observed. Sander et al. (2012) have shown that the same problem exists for Galactic WC stars. This "WR radius problem" has often been attributed to the radially extended photospheres of WR stars due to their optically thick stellar winds. However, as the WR wind extension is included in the models used for the spectral analyses above, this argument is most likely wrong and the radius problem persists. The discrepancy between the observed and theoretically predicted WR radii reaches in some cases factors up to 10 ! 


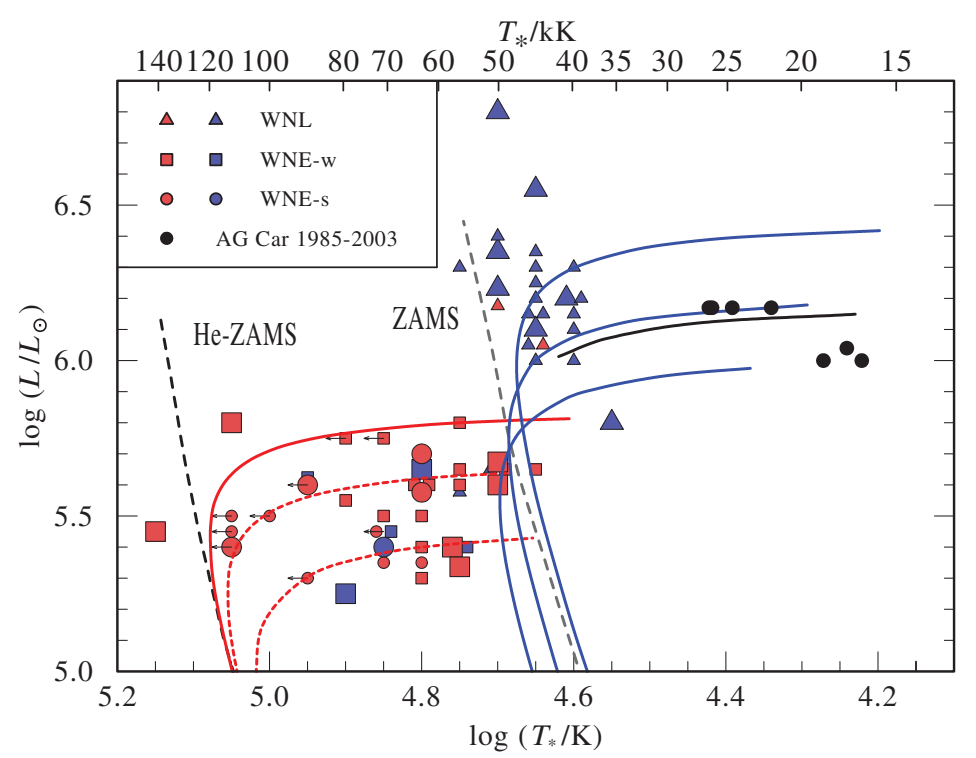

Figure 1. Hertzsprung-Russell diagram of the Galactic WN stars, and the LBV AG Car. Red/blue symbols indicate observed HR diagram positions of WN stars from Hamann et al. (2006) (blue: with hydrogen $(X>0.05)$; red: hydrogen-free $(X<0.05))$. Black symbols indicate the HR diagram positions of AG Car throughout its S Dor Cycle from 1985-2003, according to Groh et al. (2009). Large symbols refer to stars with known distances from cluster/association membership. The symbol shapes indicate the spectral subtype (see inlet). Arrows indicate lower limits of $T_{\star}$ for stars with strong mass loss. The observations are compared to stellar structure models from Gräfener et al. (2012a) (blue with hydrogen, red hydrogen-free), and for AG Car $(X=0.36$, black line). The dashed red lines indicate models for which clumping factors of 4 and 16 have been assumed in the sub-photospheric layers.

To investigate this effect Gräfener et al. (2012a) performed stellar structure computations for chemically homogeneous stars with and without hydrogen. In agreement with previous works (Ishii et al. 1999; Petrovic et al. 2006), their models show a substantial envelope inflation at high luminosities (red solid line in Fig. 1). However, to reproduce the observed WR radii it is necessary to invoke the inflation effect already at lower luminosities. Gräfener et al. managed to bring the observed WR radii in agreement with the observations by assuming that the sub-photospheric layers of WR stars are inhomogeneous, or clumped (red dashed lines in Fig. 1). Due to the higher density within clumps, clumping leads to an increased mean opacity. The adopted clumping factors are comparable with those observed in the winds of WR stars (cf. Hamann \& Koesterke 1998), hinting at a possible sub-photospheric origin of WR wind-clumping (cf. also Cantiello et al. 2009).

The underlying reason for the envelope inflation lies in the topology of the Fe-opacity peak near $\sim 160 \mathrm{kK}$. As the star has to avoid a super-Eddington situation in its subphotospheric layers, the opacity $\chi(\rho, T)$ near the Fe-peak needs to be lowered. The only way to do this for given $T$, is to go to low densities $\rho$. Close to the Eddington limit, the topology of the opacity peak thus leads to the formation of a low-density stellar envelope near $160 \mathrm{kK}$. Due to the relation between temperature and optical depth in the stellar interior (approximately with $T^{4} \propto \tau$ ), and $\tau \propto \rho \Delta R$, a low density automatically implies a large radial extension $\Delta R$. Above the low-density zone the density increases again, so that a cavity is formed (cf. Fig. 2). Gräfener et al. (2012a) could describe this effect in an analytical approach which revealed the existence of a stability limit. In this approach the 


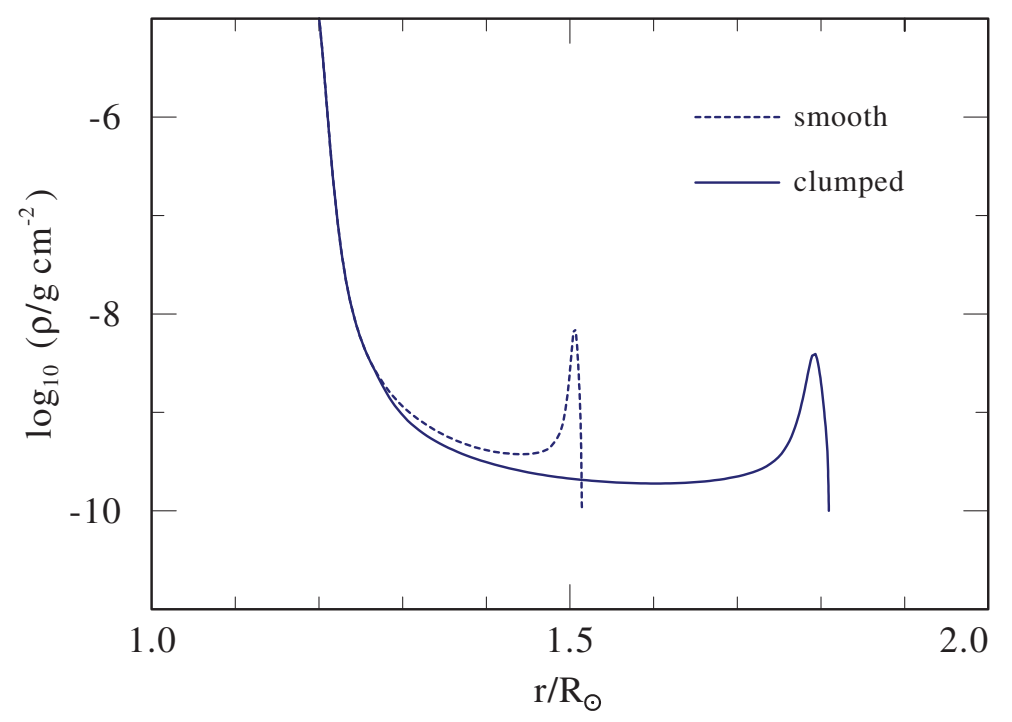

Figure 2. Density structure of inflated stellar envelopes with and without clumping from Gräfener et al. (2012a).

radial extension of the inflated layers increases with increasing stellar mass, approaching infinity at the stability limit. For higher masses no hydrostatic solutions exist.

Due to the low densities within the inflated layers the gas pressure falls far below the radiation pressure (note that the radiation pressure is a given quantity for the given Fepeak temperature). In linear stability analyses the resulting radiation-dominated cavities have been found to be subject to strange-mode pulsations which are believed to be oscillation modes that are trapped within the cavities (e.g. Saio et al. 1998). The main characteristic of these pulsations, that the pulsation period increases with increasing mass, is a direct consequence of the increasing envelope extension for increasing mass.

In non-linear simulations Glatzel \& Kaltschmidt (2002); Glatzel (2008, 2009) showed that the inflated layers may be subject to strange-mode instabilities. If these instabilities are realized in nature, they will introduce a highly inhomogeneous density structure in the inflated layers. In the same way as in the simplified clumping approach of Gräfener et al. (2012a), these inhomogeneities will lead to an enhanced mean opacity and envelope inflation. This has been illustrated in Fig. 3 of Glatzel (2009), where they compare the radial extension of a smooth hydrostatic model with one of their dynamic models. In Fig. 2 we show a very similar case where we compare a smooth and a clumped envelope model from Gräfener et al. (2012a). The resulting envelope structure is very similar to Fig. 3 in Glatzel (2009) in the sense that the clumped model shows a lower minimum density and a larger envelope extension.

The inflation effect is expected to be highly dependent on metallicity (Petrovic et al. 2006), in notable agreement with the fact that late WR subtypes are tendentially found in high-metallicity environments, such as the Galactic center, while early subtypes dominate in low-metallicity environments such as the LMC.

Another important aspect is the potential role that the envelope inflation may play for more H-rich stars near the Eddington limit. In Fig. 1 we show a comparison of our stellar structure models with the LBV AG Car during its S-Dor cycle (Groh et al. 2009). According to our computations, this star is expected to be subject to a substantial radius inflation, and is located near the discussed stability limit. This may potentially explain the nature of the erratic radius variations that are typical for LBVs. 


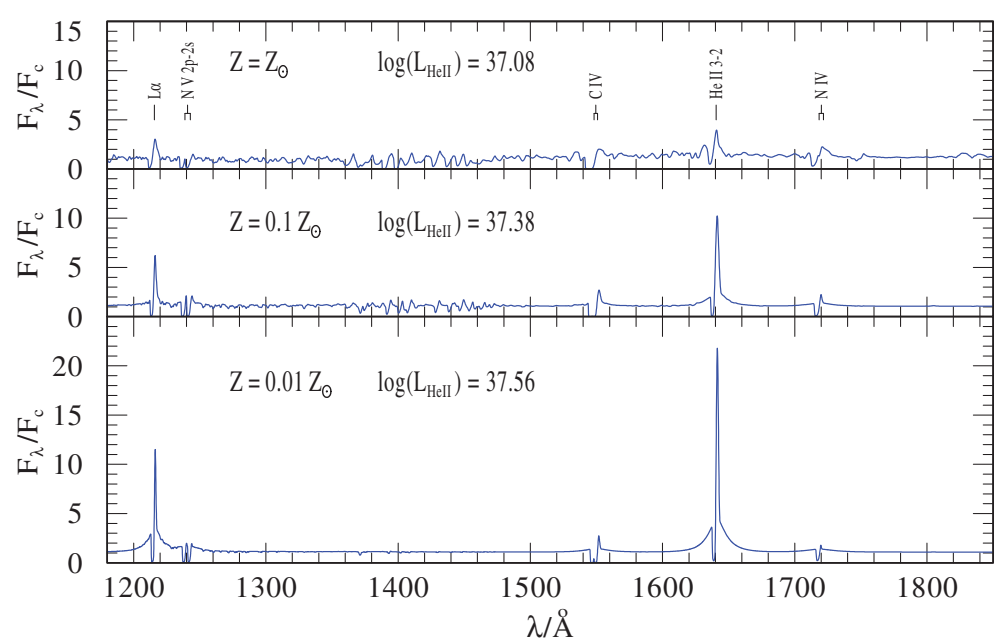

Figure 3. Synthetic UV spectra from hydrodynamically self-consistent wind models for WNh stars at different metallicities $Z$ from Gräfener \& Hamann (2008).

We note that the inflation effect is only expected to occur if convective energy transport is inefficient. Moreover, Petrovic et al. (2006) discussed that high wind mass-loss rates can destroy the effect. We also expect that the porosity of clumped material within the envelope reduces the effect. Furthermore, the inflation effect does not seem to be observed for the group of very massive WNh stars in the Galaxy (indicated by blue triangles in Fig. 1), and the LMC (cf. Bestenlehner et al. 2014). It thus seems that further dedicated studies are necessary to understand in which regime this important effect is realised in nature. Modelling and analysis of the pulsational properties of objects near the Eddington limit may be the key to resolve this question.

\section{Enhanced mass loss near the Eddington limit}

In addition to the peculiarities in their radii, WR stars and LBVs both show enhanced mass loss through stellar winds. According to our work, both phenomena are most likely related to their proximity to the Eddington limit. E.g., for very massive stars Gräfener \& Hamann (2008); Vink et al. (2011) predicted a strong mass-loss dependence on the classical Eddington factor $\Gamma_{\mathrm{e}}=\chi_{\mathrm{e}} L /(4 \pi c G M)$, where $\chi_{\mathrm{e}}$ denotes the electron scattering opacity and the other variables have their usual meaning. This dependence provides a natural explanation why these stars display WR-type spectra, although they are still burning hydrogen in their cores. The $\Gamma_{\mathrm{e}}$-dependence has been qualitatively confirmed by empirical studies of samples of very massive stars by Gräfener et al. (2011); Bestenlehner et al. (2014).

In the following we wish to highlight the importance of this effect for stellar populations at low metallicity $(Z)$. In this regime high effective values of $\Gamma_{\mathrm{e}}$ are expected because rotating massive stars tend to approach critical rotation more easily (e.g. Meynet \& Maeder 2002). Gräfener \& Hamann (2008) predicted that stars of this type can form strong WR-type winds with low terminal wind speeds. In Fig. 3 we show synthetic spectra from Gräfener \& Hamann (2008) that demonstrate that stars of this kind can form strong and narrow He II 1640 emission lines at low $Z$. Similar narrow He II emissions have recently been detected in a sample of star-forming galaxies with redshifts between 2 and 4.6 by Cassata et al. (2013). They interpreted them as nebular emission excited by a population 
of very hot Pop III stars formed in pockets of pristine gas at these redshifts. Gräfener et al. (in prep) discuss that stellar emission from a population very massive WNh stars at low $Z$ may provide an alternative explanation.

\section{References}

Bestenlehner, J. M., Gräfener, G., Vink, J. S., et al. 2014, A\&\&A, submitted

Cantiello, M., Langer, N., Brott, I., et al. 2009, A\& $A$ 499, 279

Cassata, P., Le Fèvre, O., Charlot, S., et al. 2013, A\&\&A 556, A68

Crowther, P. A., Schnurr, O., Hirschi, R., et al. 2010, MNRAS 408, 731

Glatzel, W. 2008, in A. Werner \& T. Rauch (ed.), Hydrogen-deficient stars, Vol. 391 of Astronomical Society of the Pacific Conference Series, p. 307, San Francisco: Astronomical Society of the Pacific

Glatzel, W. 2009, Communications in Asteroseismology 158, 252

Glatzel, W. \& Kaltschmidt, H. O. 2002, MNRAS 337, 743

Gräfener, G. \& Hamann, W.-R. 2008, A\&A 482, 945

Gräfener, G., Owocki, S. P., \& Vink, J. S. 2012a, A $₫ A$ 538, A40

Gräfener, G., Vink, J. S., de Koter, A., \& Langer, N. 2011, A\& A 535, A56

Gräfener, G., Vink, J. S., Harries, T. J., \& Langer, N. 2012b, A\&A 547, A83

Groh, J. H., Hillier, D. J., Damineli, A., et al. 2009, ApJ 698, 1698

Hamann, W.-R., Gräfener, G., \& Liermann, A. 2006, A\&A 457, 1015

Hamann, W.-R. \& Koesterke, L. 1998, A\&SA 335, 1003

Ishii, M., Ueno, M., \& Kato, M. 1999, PASJ 51, 417

Meynet, G. \& Maeder, A. 2002, AESA 390, 561

Petrovic, J., Pols, O., \& Langer, N. 2006, A\&A 450, 219

Saio, H., Baker, N. H., \& Gautschy, A. 1998, MNRAS 294, 622

Sander, A., Hamann, W.-R., \& Todt, H. 2012, A\&A 540, A144

Vink, J. S., Muijres, L. E., Anthonisse, B., et al. 2011, A\&A 531, A132

\section{Discussion}

MoravveJI: The position of the Fe-bump is at $\log (T / K) \sim 5.2$. Is there a possibility from theoretical calculations - that the bump is situated closer to the surface or towards the core?

GrÄFEnER: No, the bound-bound opacity calculations seem robust and there seems not much freedom from theory to change the position of the iron bump.

DE KoTER: You discussed that the clumping properties that you require in the envelope near the peak iron bump (at $150000 \mathrm{~K}$ ) are similar to the clumping properties in the outflows (the wind). Are there reasons to expect that these clumping properties should be the same?

GRÄFENER: In a recent work (Gräfener \& Vink 2013) we discussed that the location of the sonic point in WR winds follows a relation that requires that the sub-surface layers of most WR stars are clumped and inflated.

HiRschI: Where would the wind start in stars with inflated envelopes?

GrÄFENER: The models presented here are static. In the dynamic case, the location of the sonic point (and thus the mass-loss rate) will likely affect the envelope inflation. 